\title{
Obituary
}

\section{In memory of Ihor Stasyuk}

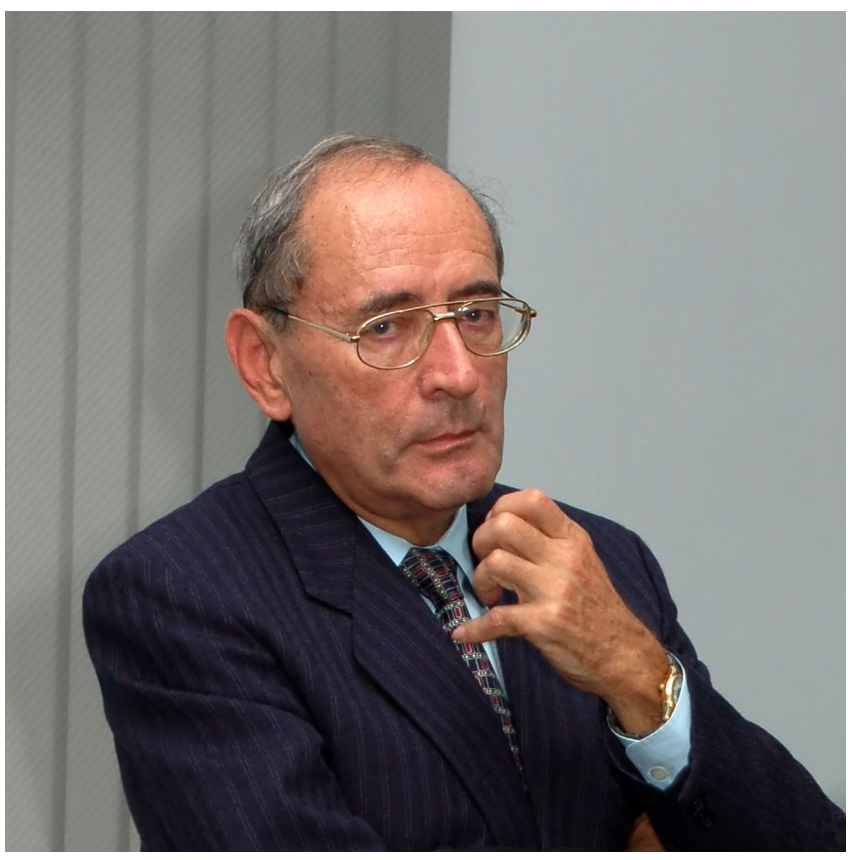

On September 16, 2019 a sudden regrettable death took the distinguished scientist Ihor Stasyuk away from us. He achieved high status within the field of theoretical physics and solid state theory: Doctor of Science in Physics and Mathematics; Professor; and Correspondent Member of the National Academy of Sciences of Ukraine.

Ihor Stasyuk was born on September 23, 1938 in the Berezhany (Ternopil region of Ukraine) to the family of a professor of mathematics. His father, Vasyl Stasyuk, graduated from the University of Vienna in 1911, where he obtained a Ph.D. in mathematics and astronomy in 1912, and very soon thereafter became a well-known mathematician and a full member of the Shevchenko Scientific Society.

Ihor Stasyuk entered the Faculty of Physics of the Ivan Franko State University of Lviv at the young age of 16 and started his research already during his student years, when, together with his supervisor Professor Abba Glauberman and V.V. Vladimirov, he developed a "new form of polar model" and introduced the "site elementary excitation" operators - predecessors of the well-known Hubbard operators. These results were acknowledged by N.N. Bogolyubov and published first in Doklady Akademii Nauk SSSR [Soviet Physics — Doklady] in 1959 and later summarized in his Ph.D. thesis "The Method of Site Elementary Excitations in the Theory of Nonmetallic Crystals," which was successfully defended in 1963. Subsequently, he became an assistant professor at the Department of Solid State Theory and later at the Department of Theoretical Physics of the same University. At that time, he obtained significant results in the theory of exchange interactions and ferromagnetism in strongly correlated electron systems, including development of the operator perturbation theory for models with local electron correlations and of Wick's theorem and diagrammatic techniques for Hubbard operators. Simultaneously, he initiated studies on the model description of dynamic and thermodynamic properties of complex hydrogen-bonded ferroelectric compounds and on the microscopic theory of optical effects in dielectric crystals.

Starting in 1978, he spent five years at the Institute of Applied Problems of Mechanics and Mathematics of the Academy of Sciences of Ukraine, where he started working on electron-deformation effects 
in semiconductors and systems with narrow bands, as well as of crystals with a cooperative Jahn-Teller effect. At that time, he developed a unified microscopic theory of the optical effects induced by external fields in dielectric crystals, including electro-gyration and the piezo-optic effect.

In 1983, Ihor Stasyuk was invited to the Lviv Division of the Institute for Theoretical Physics (ITP) of the Academy of Sciences of Ukraine, now the Bogolyubov Institute for Theoretical Physics (BITP) of the National Academy of Sciences of Ukraine (NASU), where in 1985, he successfully defended his Doctor of Science thesis entitled "Theory of External Field Induced Effects in Crystals with Structural Phase Transitions". In 1986, he became the Head of the Quantum Statistics Department. In 1990, the Lviv Division of the ITP was transformed into the Institute for Condensed Matter Physics (ICMP) and starting from that time Ihor Stasyuk was the Research Deputy Director of ICMP until he stepped down in 2006. In 1995, Ihor Stasyuk was elected a Corresponding Member of the National Academy of Sciences of Ukraine.

Professor Ihor Stasyuk completed his most important scientific achievements in the field of theoretical condensed matter physics. He is widely recognized for developing mathematical methods of the theory of multilevel systems and for his research on fermionic systems with strong short-range correlations and physical phenomena in crystals with phase transitions. Professor Stasyuk investigated the effect of Hubbard-like correlations and anharmonicity in the theory of high-temperature superconductivity and he was one of the authors of kinematic mechanisms of superconducting pairing in the Hubbard model. The school of Professor Stasyuk is widely acknowledged for the development of a microscopic theory for different types of field effects (hydrostatic and uniaxial pressure, electric field, single-ion anisotropy, etc.) and on the properties and thermodynamics of ferroelectric and Jahn-Teller crystals. Another field of interest was connected with protonic and ionic transport in systems with superionic phases. During his last years, Stasyuk's interests concerned the theory of intercalation induced effects in crystals and phase transitions in optical lattices. His last article, which considers Bose-Einstein condensation in the Bose-Fermi-Hubbard model, is published in this issue. Over his long-lasting career, Prof. Stasyuk has authored six books and more than 700 scientific papers and contributions.

In addition to his research career, Professor Ihor Stasyuk was also well-known as an outstanding teacher. He always combined his research work with his educational activities. Twenty one researchers under his supervision received Ph.D. degrees; five of them have already become Doctors of Science. Numerous generations of students of the Ivan Franko National University of Lviv remember his brilliant lectures in theoretical physics, as well as his more specific courses in quantum statistics, electrodynamics, solid state theory, phase transitions theory, mathematical methods in theoretical physics, and many more.

Professor Ihor Stasyuk was an active organizer of science. He was a full member of the Shevchenko Scientific Society, associate editor of "Condensed Matter Physics", editor of many international journals and member of the International Advisory Committee on Ferroic Domains and Mesoscopic Structures. For many years he was the vice-president of the Ukrainian Physical Society. Ihor Stasyuk also served as either the conference chair or as a member of the organizing and programme committee of many international and Ukrainian conferences in physics.

His scientific and public activity was acknowledged by many awards and titles. Professor Ihor Stasyuk was the Soros Professor (1996) and the "Excellence in Education of Ukraine" badge holder (1998). He was awarded by the Certificate of Honour of the Supreme Council of Ukraine in 2004 and the Insignia "For Scientific Achievements" of the National Academy of Sciences of Ukraine in 2008. I.V.Stasyuk is the Chevalier of the Order of Merit of Third (2009) and Second (2018) Classes. In 2011, he was elected a Doctor Philosophiae Honoris Causa at the Bogolyubov Institute for Theoretical Physics and in 2014, he was awarded the A.S. Davydov Prize of the National Academy of Sciences of Ukraine.

With Ihor Stasyuk's sudden passing, the community of condensed matter physics has lost a unique theoretical physicist. However, his influence lives on in everybody who was has had the pleasure to interact with him. Ihor Stasyuk won the respect and appreciation of his colleagues and friends by his polite, attentive, and kind way-traits specific to his warm personality. He will be remembered as an accomplished scientist, a devoted mentor, a gifted author, and a good friend. He will be missed by all.

The Editorial Board of "Condensed Matter Physics" present our condolences to the family of Ihor Stasyuk and his numerous colleagues and friends in connection with his untimely death.

Jim Freericks, Yurij Holovatch, Olexandre Ivankiv, Ihor Mryglod, Oksana Patsahan, Andrij Shvaika 